\title{
Improvement of deep cross-modal retrieval by generating real-valued representation
}

\author{
Nikita Bhatt ${ }^{\text {Corresp., } 1}$, Amit Ganatra ${ }^{2}$ \\ 1 U \& P U.Patel Department of Computer Engineering, Chandubhai S Patel Institute of Technology, Anand, Gujarat, India \\ 2 Devang Patel Institute of Advance Technology and Research, Anand, Gujarat, India \\ Corresponding Author: Nikita Bhatt \\ Email address: nikitabhatt.ce@charusat.ac.in
}

The Cross-Modal Retrieval (CMR) has attracted much attention in the research community due to flexible and comprehensive retrieval. The core challenge in CMR is the heterogeneity gap, which is generated due to different statistical properties of Multi-Modal data. The most common solution to bridge the heterogeneity gap is representation learning, which generates a common sub-space. In this work, we propose a framework called "Improvement of Deep Cross-Modal Retrieval (IDCMR)", which generates real-valued representation. The IDCMR preserves both intra-modal and inter-modal similarity. The intra-modal similarity is preserved by selecting an appropriate training model for text and image modality. The inter-modal similarity is preserved by reducing modality-invariance loss. The Mean Average Precision (mAP) is used as a performance measure in the CMR system. Extensive experiments are performed, and results show that IDCMR outperforms over state-of-the-art methods by a margin $4 \%$ and $2 \%$ relatively with mAP in the text to image and image to text retrieval tasks on MSCOCO and Xmedia dataset respectively. 
1 Improvement of deep cross-modal retrieval by

2 generating real-valued representation

3

4

5

6

7 10

\author{
Nikita Bhatt ${ }^{1}$, Amit Ganatra ${ }^{2}$ \\ ${ }^{1}$ U \& P U Patel Department of Computer Engineering, Chandubhai S Patel Institute of \\ Technology, CAHRUSAT, Anand, Gujarat, India \\ 2 Principal, Devang Patel Institute of Advance Technology and Research, Anand, Gujarat, India
}

Corresponding Author:

Nikita Bhatt ${ }^{1}$

U \& P U Patel Department of Computer Engineering, Chandubhai S Patel Institute of

Technology, CAHRUSAT, Anand, 388421, Gujarat, India

Email address: nikitabhatt.ce@charusat.ac.in

\title{
Abstract
}

The Cross-Modal Retrieval (CMR) has attracted much attention in the research community due to flexible and comprehensive retrieval. With different statistical properties of Multi-Modal data, a heterogeneity gap is generated, which does not allow a direct comparison for retrieval. The most common solution to bridge the heterogeneity gap is representation learning, which generates a common sub-space from different modalities. The state-of-the-art CMR methods use hashing techniques to generate binary-valued representation in the common sub-space however, it suffers from information loss. To resolve the problem of information loss, we propose a framework called "Improvement of Deep Cross-Modal Retrieval (IDCMR)", which generates real-valued representation. The real-valued representation stores actual information of different modalities by preserving similarity between them. The objective function of the proposed framework preserves inter-modal and intra-modal similarities between different modalities. Further, the intra-modal similarity is preserved by selecting an appropriate training model for each modality. The biggest challenge in text modality is to preserve similarity between different words. There are many vectorization methods available, which map words into vectors. However, the selection of an appropriate method is a challenging task. Here experiments are performed using various vectorization methods on Multi-Modal datasets. Further, extensive experiments are performed on the proposed framework, and performance is compared with stateof-the-art methods. The results presented in the paper show that IDCMR outperforms state-ofthe-art methods by a margin of $4 \%$ and $2 \%$ relatively with mean average precision (mAP) in the text $\rightarrow$ image and image $\rightarrow$ text retrieval tasks on MSCOCO and XMedia dataset respectively. 


\section{Introduction}

41 In the era of big data, multimedia data such as text, image, audio, and video are growing at an unprecedented rate. Such Multi-Modal data has enriched people's lives and become a

44

45

46

47

48

49

50

51

52

53

54

55

56

57

58

59

60

61

62

63

64

65

66

67

68

69

70

71

72

73

74

75

76

77

78

79 fundamental component to understand the real world. We access Multi-Modal data in various situations like education, entertainment, advertisements, social media, which are helpful to provide effective communication. Also, real-world articles use different modalities to provide comprehensive information about any concept or topic. In recent years, Image Captioning and Cross-Modal Retrieval (CMR) have become hot research directions in vision-language tasks (Xu, Li \& Zhang, 2020; Yanagi et al., 2020). The difference between them is shown in Figure 1. The Image Captioning system, as shown in Figure 1(A) and Figure 1(B), takes an image from the MSCOCO dataset (Lin et al., 2015b) and retrieves the description of an image in the form of text. Here the retrieved information is provided by both modalities (e.g. the word "cat" and pixels of "cat" are closed to each other in a learning space). On the other hand, the CMR system provides flexible retrieval where the user can give any modality as the input and retrieves any other modality as the output. As shown in Figure 1(C) and Figure 1(D), an image of "owl" from the XMedia dataset ("PKU XMediaNet Dataset")can be explained with multiple texts, which is not visible from the image only. Such comprehensive retrieval from the CMR system is widely used in applications like hot topic detection and personalized recommendation(Peng et al., 2017). The real challenge in CMR is the heterogeneity gap (Wang et al., 2016b), which is generated due to the different statistical properties of each modality. For example, an image representation is real-valued and dense in the form of pixels, whereas text representation is sparse and discrete. Such a different representation of each modality does not allow a direct comparison for retrieval. The most common solution to bridge the heterogeneity gap is to generate a common sub-space (Zhen et al., 2019) using a function, which transforms the different representation of modalities into a common representation, such that direct retrieval is possible. Many approaches for CMR have been proposed in the past to generate a common sub-space, which is categorized into binary-valued and real-valued representation. The binary-valued representation maps heterogeneous data into the encoded form using a hash function. The advantage of binary-valued representation is less storage, which leads to faster retrieval because hamming distance can be computed faster with the help of binary code using bit operations. However, binary-valued representation suffers from information loss, which leads to unsatisfactory performance. In this paper, real-valued representation is considered, which stores actual representation. Previous CMR methods like spectral hashing (SH) (Weiss, Torralba \& Fergus, 2009), cross-view hashing(CVH)(Kumar \& Udupa, 2011), Inter-Media Hashing (IMH)(Song et al., 2013), Collective Matrix Factorization Hashing (CMFH) (Ding, Guo \& Zhou, 2014), Semantic Correlation Maximization (SCM)(Zhang \& Li, 2014), Latent semantic sparse hashing (LSSH) (Zhou, Ding \& Guo, 2014) and Semantic Preserving Hashing (SePH) (Lin et al., 2015a) perform feature learning and correlation learning as an independent process to generate a common subspace. All these CMR methods perform feature learning using scale-invariant feature transform (SIFT) (Lowe, 2004) and histogram of oriented gradients (HoG) (Hardoon, Szedmak \& Shawe- 
80 Taylor, 2004). However, the correlation learning ignores the correlation between different

81 modalities during feature learning, which may not achieve satisfactory performance. The

82 standard statistical correlation-based method is Canonical Correlation Analysis (CCA) (Hardoon,

83 Szedmak \& Shawe-Taylor, 2004), which learns linear projections from heterogeneous data, and

84 a common sub-space is generated. However, Multi-Modal data is involved with non-linear

85 relations, which cannot be learned with CCA. So, some kernel-based approach(Hongtao \&

86 Qinchuan, 2016) has been proposed which can handle the problem, but the selection of the

87 kernel function is one of the open challenges.

88 Motivated with great power and success of deep learning in the domain of representation

89 learning, a variety of approaches have been proposed, which generates a common sub-space. The

90 work presented in (Ngiam et al., 2011), proposes a deep auto-encoder (DAE) to learn the

91 correlation between Multi-Modal data and a Restricted Boltzmann Machine (RBM) to learn a

92 common sub-space in an unsupervised way. In (Srivastava \& Salakhutdinov, 2014), a graphical-

93 based model called deep Boltzmann machine (DBM) is used which does not need supervised

94 data for training, and each layer of the Boltzmann machine adds more level of abstract

95 information. In (Jiang \& Li, 2016), a framework called Deep Cross-Modal Hashing (DCMH) is

96 proposed, which generates a common sub-space in a supervised way, and similarity is preserved

97 by forcing image and text representation to be as close as possible. In (Wang et al., 2016a),

98 Convolutional Neural Network (CNN) for image modality and Neural Language Model for text

99 modality is used to learn a common sub-space using a mapping function. The Euclidean distance

100 calculates the distance between image and text representation, which is useful for Cross-Modal

101 learning. In (Zhen et al., 2019), a framework called Deep Supervised Cross-Modal Retrieval

102 (DSCMR) is proposed, which uses CNN for image modality and word2vec for text modality,

103 which generates real-valued representation. A lot of work is carried out in CMR, but the

104 performance of the CMR system can be further improved by maintaining both intra-modal and

105 inter-modal similarity as much as possible. In this paper, we propose a novel framework called

106 "Improvement of Deep Cross-Modal Retrieval (IDCMR)", which generates a common sub-space

107 by preserving similarity between image and text modality. The objective function of IDCMR

108 preserves both inter-modal and intra-modal similarity. The main contributions of IDCMR are

109 summarized as follow:

110

111

- The proposed framework IDCMR performs feature learning and correlation learning in the same framework.

112

113

- Our proposed framework preserves intra-modal semantic similarity for text modality.

114

115

116

117 Experiments are performed using various vectorization methods on Multi-Modal datasets for the selection of an appropriate vectorization method for text modality.

- The IDCMR generates real-valued representation in the common sub-space, which preserves inter-modal and intra-modal similarities between image and text modality.

118

- The Mean Average Precision (mAP) is used as a performance measure, and a comparison of the proposed framework is made with state-of-the-art methods. 
119 The rest of the paper is divided as follows. Section 2 gives the background of vectorization 120 methods for text modality. Section 3 covers the proposed model and the proposed algorithm.

121 Section 4 covers experiments and discussion. At last, Section 5 gives the conclusion of our work.

\section{Background and Literature Survey}

123 The biggest challenge in Natural Language Processing (NLP) is to design algorithms, which

124

125

126

127

128

129

130

131

132

133

134

135

136

137

138

139

140

141

142

143

144

145

146

147

148

149

150

151

152

153

154

155

156 allows computers to understand natural language to perform different tasks. It is recommended to represent each word in form of a vector as most of the machine learning algorithms are not capable of processing text directly in its raw form. The process of converting a word into a vector is called vectorization, which represents each word into vector space. Broadly the vectorization methods are categorized into (a) local representation method and (b) distributional representation method. The most common local representation method is called bag-of-words (BoW), where each word is represented as $\mathbb{R}^{|V| \times 1}$ vector with all 0 's and one 1 at the index of the word in the corpus. However, the generated matrix is sparse in nature, which is inefficient for computation, and the similarity between different words is not preserved, as the inner product between two different one-hot vectors is zero. On the other hand, in distributional representation, each word $\mathrm{w}_{\mathrm{i}}$ in the corpus is represented by featurized representation, which is denoted as $\mathrm{w}_{\mathrm{i}} \in$ $\mathcal{R}^{\mathrm{d}}$, where each word is represented in d dimensions.

The distributional representation generates distributional word vectors, which follows the concept of the distributional hypothesis (Mikolov et al., 2013b), which states that words that occur in the same contexts tend to have similar meanings. The distributional word vectors are generated from count-based models or prediction based models. The count-based models generate implicit distributional vectors using dimensionality-reduction techniques, which map data in the high-dimensional space to a space of fewer latent dimensions. The most popular method is Singular Value decomposition (SVD) (Van Loan, 1976), which generates embedding of each word in the vocabulary using matrix factorization, but fails when the dimensionality of matrices is very large as the computational cost for $\mathrm{m} \times \mathrm{n}$ matrix is $\mathrm{O}\left(\mathrm{mn}^{2}\right)$. The most popular count-based method is Glove(Mikolov et al., 2013b), which generates implicit vector and achieve better performance in comparison with other matrix-based methods. Another broader classification for the generation of distributional word vector is prediction based models, which are neural network based algorithms. Such models directly create low-dimensional implicit distributional representations. An example of such a model is word2vec. The below section covers a detailed description of the generation of word vectors using Glove and word2vec.

\section{word2vec}

The word2vec is a feed-forward based neural network, which has two algorithms: continuous bag-of-words (CBOW) and skip-gram (SG) (Mikolov et al., 2013a,b). Figure 2 (Mikolov et al., 2013a) shows a description of CBOW and SG where CBOW predicts the probability of center word $w(t)$ and $S G$ predicts the probability of surrounding words $w(t+j)$.

\section{Working of SG model}


157 SG predicts the probability of surrounding given a center word. For training of the network, there 158 is an objective function that maximizes the probability of surrounding words given center word 159 for each position of text $t$ in the window size of $\mathrm{m}$.

$$
J^{\prime}(\theta)=\prod_{t=1}^{T} \prod_{\substack{-m_{j} \leq j \leq m \\ j \neq 0}} P\left(w_{t+j} \mid w_{t} ; \theta\right)
$$

160 Here $P\left(w_{t+j} \mid w_{t}\right)$ is probability of surrounding words $w_{t+j}$ given center word $w_{t}$. Eq. (1) can 161 be rewritten as equation (2)

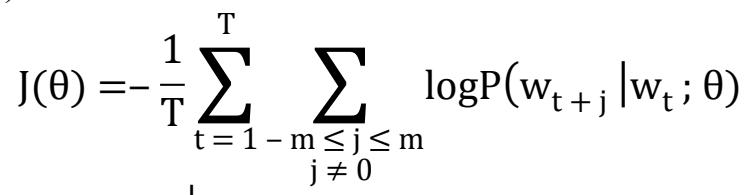

$162 \mathrm{P}\left(\mathrm{w}_{\mathrm{t}+\mathrm{j}} \mid \mathrm{w}_{\mathrm{t}}\right)$ can be rewritten as $\mathrm{P}(\mathrm{o} \mid \mathrm{c})$, which specifies the probability of surrounding words $\mathrm{o}$ 163 given center word c, and softmax function is used to generate probability.

$$
P(o \mid c)=\frac{e^{u_{0}^{T} v_{c}}}{\sum_{w=1}^{v} e^{u_{w}^{T} v}}
$$

164 Where $\mathrm{u}_{0}$ specifies vector representation of the surrounding word at index 0 and $\mathrm{v}_{\mathrm{c}}$ specifies the 165 vector representation of center word. Equation (3) can be applied in equation (2),

$$
J(\theta)=\log \frac{e^{u_{0}^{T} v_{c}}}{\sum_{w=1}^{v} e^{u_{w}^{T} v_{c}}}
$$

166 Now, the objective is to optimize $\mathrm{v}_{\mathrm{c}}$ and $\mathrm{u}_{\mathrm{w}}$. So need to take the derivative with respect to $\mathrm{v}_{\mathrm{c}}$ and $167 \mathrm{u}_{\mathrm{w}}$.

$$
J(\theta)=\frac{\partial}{\partial v_{c}} \log e^{u_{0}^{T} v_{c}}-\frac{\partial}{\partial v_{c}} \log \sum_{w=1}^{v} e^{u_{w}^{T} v_{c}}
$$

Where,

$$
\begin{gathered}
\frac{\partial}{\partial \mathrm{v}_{\mathrm{c}}} \log \mathrm{e}^{\mathrm{u}_{0}^{\mathrm{T}} \mathrm{v}}=u_{0} \\
\frac{\partial}{\partial \mathrm{v}_{\mathrm{c}}} \log \sum_{\mathrm{w}=1}^{\mathrm{v}} \mathrm{e}^{\mathrm{u}_{\mathrm{w}}^{\mathrm{T}} \mathrm{v}}=\frac{1}{\sum_{\mathrm{w}=1}^{\mathrm{v}} \mathrm{e}^{\mathrm{u}_{\mathrm{w}}^{\mathrm{T}} \mathrm{v}}} \times \frac{\partial}{\partial \mathrm{v}_{\mathrm{c}}} \sum_{\mathrm{w}=1}^{\mathrm{v}} \mathrm{e}^{\mathrm{u}^{\mathrm{T}} \mathrm{w}^{\mathrm{v}} \mathrm{c}}
\end{gathered}
$$




$$
\begin{aligned}
& =\frac{1}{\sum_{w=1}^{v} e^{u_{w}^{T} v_{c}}} \times \frac{\partial}{\partial v_{c}} \sum_{x=1}^{V} e^{u_{x}^{T} v_{c}} \\
& =\frac{1}{\sum_{w=1}^{\mathrm{V}} \mathrm{e}^{\mathrm{u}_{\mathrm{w}}^{\mathrm{T}} \mathrm{v}}} \times \sum_{\mathrm{x}=1}^{\mathrm{V}} \frac{\partial}{\partial \mathrm{v}_{\mathrm{c}}} \mathrm{e}^{\mathrm{u}_{\mathrm{x}}^{\mathrm{T}} \mathrm{v}_{\mathrm{c}}} \\
& =\frac{1}{\sum_{w=1}^{v} e^{u_{w}^{T} v_{c}}} \times \sum_{x=1}^{V} e^{u_{x}^{T} v_{c} \frac{\partial}{\partial v_{c}}} e^{u_{x}^{T} v_{c}} \\
& =\frac{1}{\sum_{w=1}^{\mathrm{v}} \mathrm{e}^{\mathrm{u}_{\mathrm{w}}^{\mathrm{T}} \mathrm{v}}} \times \sum_{x=1}^{V} \mathrm{e}^{\mathrm{u}_{\mathrm{x}}^{\mathrm{T}} \mathrm{c}} u_{x}
\end{aligned}
$$

Combine (5.a) and (5.b),

$$
J(\theta)=u_{0}-\frac{1}{\sum_{w=1}^{v} e^{u_{w}^{T} v_{c}}} \times \sum_{x=1}^{v} e^{u_{x}^{T} v^{T} c} u_{x}
$$

Above equation can be rewritten as

$$
J(\theta)=u_{0}-\sum_{x=1}^{V} P(x \mid c) \cdot u_{x}
$$

168 Where, $\mathrm{u}_{0}$ is the actual ground truth and $\mathrm{P}(\mathrm{x} \mid \mathrm{c})$ is the probability of each surrounding word $\mathrm{x}$ given the center word $c$, and $u_{x}$ is the average of all possible surrounding words. So cost function of SG guarantees that the probability of occurring surrounding words maximizes given a center word.

\section{Working of continuous bag-of-words model} CBOW predicts the probability of a center word given surrounding words. Input to CBOW is $\mathrm{d}$ dimensional one-hot vector representation of a center word. The representation of a center word is generated by multiplying $d$ dimensional vector with the weight matrix $W$ of size $p \times d$ where $p$ is the featurized representation of a word.

$$
\mathrm{h}_{\mathrm{p} \times 1}=\mathrm{W}_{\mathrm{p} \times \mathrm{d}^{\mathrm{T}}}^{\mathrm{T}} \mathrm{x}_{\mathrm{c}}=\mathrm{V}_{\mathrm{c}}
$$

177 The above representation is a vector representation of the center word $\mathrm{V}_{\mathrm{c}}$. The representation of

178 outside words is generated by multiplying center representation with the weight matrix W'. 


$$
\mathrm{u}_{\mathrm{d} \times 1}=\mathrm{W}_{\mathrm{d} \times \mathrm{p}}^{\mathrm{T}} \mathrm{h}_{\mathrm{p} \times 1}=\mathrm{V}_{\mathrm{w}}^{\mathrm{T}} \mathrm{V}_{\mathrm{c}}
$$

179 Where, $V_{c}$ is a vector representation of the center word and $V_{w}$ is a vector representation of 180 surrounding words. It is a prediction-based model so need to find the probability of a word given 181 the center word $\mathrm{P}(\mathrm{w} \mid \mathrm{c})$.

$$
y_{i}=P(w \mid c)=\varphi\left(u_{i}\right)=\frac{e^{u_{i}}}{\sum_{i^{\prime}} e^{u_{i}}}=\frac{e^{\mathrm{V}_{w^{T}}^{T} v_{c}}}{\sum_{w^{\prime} \in \text { Text }} e^{\mathrm{V}_{w^{\prime}}{ }^{\prime} v_{c}}}
$$

182 There is an objective function, which maximizes $\mathrm{P}(\mathrm{w} \mid \mathrm{c})$ by adjusting the hyper parameters i.e., $\mathrm{v}_{\mathrm{c}}$ 183 and $\mathrm{v}_{\mathrm{w}}$.

$$
l(\theta)=\sum_{w \in \text { Text }} \log P(w \mid c ; \theta)
$$

184 Put value of equation (9) in equation (10),

$$
\begin{aligned}
& l(\theta)=\sum_{w \in \text { Text }} \log \frac{e^{v_{w}^{T} v_{c}}}{\sum_{w^{\prime} \in \text { Text }} e^{v_{w^{\prime} v_{c}}^{T}}} \\
& =\sum_{w \in \text { Text }} \log \mathrm{e}^{\mathrm{v}_{\mathrm{w}}^{\mathrm{T}} \mathrm{v}_{\mathrm{c}}}-\sum_{\mathrm{w} \in \mathrm{Text}} \log \frac{1}{\sum_{\mathrm{w}^{\prime} \in \text { Text }} \mathrm{e}^{\mathrm{v}_{\mathrm{w}^{\prime} \mathrm{v}}^{\mathrm{T}} \mathrm{v}}}
\end{aligned}
$$

185 To optimize the hyper parameter, need to take derivation with respect to $\mathrm{v}_{\mathrm{c}}$ and $\mathrm{v}_{\mathrm{w}}$.

$$
\frac{\partial l}{\partial \mathrm{v}_{\mathrm{w}}}=\sum_{\mathrm{w} \in \mathrm{Text}} \mathrm{v}_{\mathrm{c}}-\frac{1}{\sum_{\mathrm{w}^{\prime} \in \text { Text }} \mathrm{e}^{\mathrm{v}^{\mathrm{T}}, \mathrm{v}_{\mathrm{c}}}} \times \frac{\partial l}{\partial w} \mathrm{e}^{\mathrm{v}_{\mathrm{w}}^{\mathrm{T}}, \mathrm{v}_{\mathrm{c}}}
$$

$$
=\sum_{w \in \text { Text }} \mathrm{v}_{\mathrm{c}}-\frac{1}{\sum_{\mathrm{w}^{\prime} \in \mathrm{Text}} \mathrm{e}^{\mathrm{v}^{\mathrm{T}}, \mathrm{v}_{\mathrm{c}}}} \times \mathrm{e}^{\mathrm{v}^{\mathrm{T}}, \mathrm{v}^{\mathrm{c}} \mathrm{c}} \times \mathrm{v}_{\mathrm{c}}
$$

$$
189=\sum_{w \in \text { Text }} \mathrm{v}_{\mathrm{c}}[1-\mathrm{P}(\mathrm{w} \mid \mathrm{c})]
$$

$$
=\sum_{w \in \text { Text }} v_{c}-P(w \mid c) v_{c}
$$

190 For optimization, gradient descent algorithm is applied and hyper parameter is optimized.

$$
\mathrm{V}_{\mathrm{w}}=\mathrm{V}_{\mathrm{w}}-\eta \mathrm{V}_{\mathrm{c}}[1-\mathrm{P}(\mathrm{w} \mid \mathrm{c})]
$$


191 Similar steps are followed for hyperparameter $V_{c}$.

$$
\mathrm{V}_{\mathrm{c}}=\mathrm{V}_{\mathrm{c}}-\eta \mathrm{V}_{w}[1-\mathrm{P}(\mathrm{w} \mid \mathrm{c})]
$$

192 So CBOW and SG preserve the semantic similarity by following the distributional hypothesis in 193 comparison with BoW model.

\section{Glove (Count Based Method)}

195 In contrast to word2vec, Glove captures the co-occurrence of a word from the entire

196 corpus(Pennington, Socher \& Manning, 2014). Glove first constructs the global co-occurrence

197 matrix $X_{i j}$, which gives information about how often words $i$ and $j$ appear in the entire corpus.

198 The size of the matrix can be minimized by the factorization process, which generates a lower199 dimensional matrix such that reconstruction loss is minimized. The objective of the Glove model 200 is to learn the vectors $v_{i}$ (vector representation of $i$ word) and $v_{j}$ (vector representation of $j$ word), 201 which are fruitful to information which is in the form of $X_{\mathrm{ij}}$. The similarity between words is 202 captured by finding the inner product $v_{i}^{T} v_{j}$, which gives similarity between words $i$ and $j$. This 203 similarity is proportional to $\mathrm{P}(\mathrm{j} \mid \mathrm{i})$ or $\mathrm{P}(\mathrm{i} \mid \mathrm{j})$, where $\mathrm{P}(\mathrm{j} \mid \mathrm{i})$ gives the probability of word $\mathrm{j}$ given the 204 word i.

205

206

$$
\mathrm{v}_{\mathrm{i}}^{\mathrm{T}} \mathrm{v}_{\mathrm{j}}=\log \mathrm{P}(\mathrm{j} \mid \mathrm{i})
$$

$$
\text { Where, } \log P(j \mid i)=\frac{X_{i j}}{\sum X_{i j}}=\frac{X_{i j}}{X_{i}}
$$

$$
\mathrm{v}_{\mathrm{i}}^{\mathrm{T}} \mathrm{v}_{\mathrm{j}}=\log \mathrm{X}_{\mathrm{ij}}-\log \mathrm{X}_{\mathrm{i}}
$$

207 Similarly,

$$
\mathrm{v}_{\mathrm{j}}^{\mathrm{T}} \mathrm{v}_{i}=\log \mathrm{X}_{\mathrm{ij}}-\log \mathrm{X}_{\mathrm{j}}
$$

208

209

Equation (15) and (16) are added,

$$
\begin{aligned}
2 \mathrm{v}_{\mathrm{j}}^{\mathrm{T}} \mathrm{v}_{i} & =2 \log \mathrm{X}_{\mathrm{ij}}-\log \mathrm{X}_{\mathrm{i}}-\log \mathrm{X}_{\mathrm{j}} \\
\mathrm{v}_{\mathrm{j}}^{\mathrm{T}} \mathrm{v}_{\mathrm{i}} & =\log \mathrm{X}_{\mathrm{ij}}-\frac{1}{2} \log \mathrm{X}_{\mathrm{i}}-\frac{1}{2} \log \mathrm{X}_{\mathrm{j}}
\end{aligned}
$$

210 Here $v_{i}$ and $v_{j}$ are learnable parameters and $X_{i}, X_{j}$ is word specific biases, which will be learned 211 as well. The above equation can be rewritten as

$$
\mathrm{v}_{\mathrm{j}}^{\mathrm{T}} \mathrm{v}_{\mathrm{i}}+\mathrm{b}_{\mathrm{i}}+\mathrm{b}_{\mathrm{j}}=\log \mathrm{X}_{\mathrm{ij}}
$$

212 Where $b_{i}$ is word specific bias for word $i$ and $b_{j}$ is word specific bias for word $j$. All these

213 parameters are learnable parameters, whereas $X_{i j}$ is the actual ground truth that can be known

214 from the global co-occurrence matrix. Eq. (16) can be formulated as an optimization problem, 215 which gives the difference between predicted value using model parameters and the actual value 216 computed from the given corpus.

$$
\min _{v_{i}, v_{j}, b_{i}, b_{j}} \sum_{i, j}\left(v_{j}^{T} v_{i}+b_{i}+b_{j}-\log X_{i j}\right)^{2}
$$


217 In comparison with word2vec, Glove maintains both the local and global context of a word from

218 the entire corpus. To select an appropriate vectorization method, which maintains intra-modal

219 semantic coherence, the below section covers experiments performed using different

220 vectorization methods on Multi-Modal datasets. The Convolutional Neural Network (CNN) is

221 adopted for image modality in the proposed framework, as it has shown promising performance

222 in many computer vision applications(Hongtao \& Qinchuan, 2016).

223

224

\section{Materials \& Methods}

225

226

227

228

229

230

231

232

233

234

235

236

237

238

239

240

241

242

243

244

245

246

247

248

249

250

251

252

253

\section{Proposed framework for cross-modal retrieval}

In this section, we present our proposed framework, which generates real-valued common subspace. It also covers the learning algorithm outlined in Algorithm: IDCMR.

\section{Problem formulation}

The proposed framework has image and text modality, which is denoted by $\Psi=\left\{\left(X_{i}, Y_{i}\right)\right\}_{i=1}^{n}$ where $X_{i}$ and $Y_{i}$ is image and text sample respectively. Each instance of $\left(X_{i}, Y_{i}\right)$ has a semantic label vector $\mathrm{Z}_{\mathrm{i}}=\left[\mathrm{z}_{1 \mathrm{i}}, \mathrm{z}_{2 \mathrm{i}}, \ldots, \mathrm{z}_{\mathrm{Ci}}\right] \in \mathrm{R}^{\mathrm{C}}$, where $\mathrm{C}$ is the number of categories. The similarity matrix $S_{i j}=1$, if $i^{\text {th }}$ instance of image and text modality matches to the $j^{\text {th }}$ category, otherwise $S_{i j}=0$. The feature vectors of image and text modality lie in different representation space, so direct composition is not possible for retrieval. The objective is to learn two functions, $\mathrm{u}_{\mathrm{i}}=\mathrm{f}\left(\mathrm{x}_{\mathrm{i}}, \theta_{\mathrm{x}}\right) \in$ $\mathbb{R}^{d}$ and $\mathrm{v}_{\mathrm{i}}=\mathrm{g}\left(\mathrm{y}_{\mathrm{i}}, \theta_{\mathrm{y}}\right) \in \mathbb{R}^{d}$ for image and text modality respectively, where $\mathrm{d}$ is the dimension of a common sub-space. The $\theta_{\mathrm{x}}$ and $\theta_{\mathrm{y}}$ are hyper parameters of image and text modality, respectively. The generated common sub-space allows direct comparison for retrieval even though samples come from different statistical properties.

\section{Proposed framework: Improvement of deep cross-modal retrieval (IDCMR)}

Figure 3 shows the proposed framework for image and text modality. The convolutional layers of Convolutional Neural Network (CNN) for image modality are pretrained on ImageNet, which generates high-level representation for each image. CNN has five convolutional layers and three fully connected layers. Detailed configuration of the convolutional layer is given in the proposed framework. Each convolutional layer contains "f: num $\times$ size $\times$ size", which specifies the number of the filter with specific size, "s" indicates stride, "pad" indicates padding, and "pool" indicates downsampling factor. The common representation for each image is generated by fully connected layers. The number in the last fully connected layer (fc8) indicates the number of neurons or dimensionality of the output layer. Similarly, the Glove model for text modality is pretrained on Google News, which represents each word in form of feature vector. The text matrix is given to fully connected layers to learn the common representation for text. To learn a common representation from image and text modality, the two sub-networks share the weights of the last layers, which generate the same representation for semantic similar image and text modality. In this work, real-valued coordinated representation is generated, which preserves 
254 intra-modal and inter-modal semantic similarity. The inter-modal similarity is preserved by

255 minimizing the (i) discrimination loss in the label space $\mathrm{J}_{1}$. The prediction of label from feature

256 spaces is possible, by connecting a linear classifier on top of each network. (ii) discrimination

257 loss in text and image representation $\mathrm{J}_{2}$, and (iii) modality-invariant loss $\mathrm{J}_{3}$ in the common sub-

258 space. Further, the intra-modal similarity is preserved by selecting an appropriate training model 259 for each modality. The biggest challenge in text modality is to preserve semantic similarities

260 between words. There are many distributional representation methods available and the

261 challenge is to select an appropriate method, which preserves intra-modal similarity between

262 different words of text modality. The below section covers the learning algorithm, experiments

263 of different distributional models, and performance comparison of the proposed framework with

264 state-of-the-art methods.

\section{Algorithm: IDCMR}

266 Input:

$267 \Psi=\left\{\left(\mathrm{X}_{\mathrm{i}}, \mathrm{Y}_{\mathrm{i}}\right)\right\}_{\mathrm{i}=1}^{\mathrm{n}}$ where $\mathrm{X}_{\mathrm{i}}$ is the input image sample, and $\mathrm{Y}_{\mathrm{i}}$ is the input text sample of ith 268 instance.

$269 \mathrm{Z}_{\mathrm{i}}=\left[\mathrm{z}_{1 \mathrm{i}}, \mathrm{z}_{2 \mathrm{i}}, \ldots ., \mathrm{z}_{\mathrm{Ci}}\right] \in \mathrm{R}^{\mathrm{C}}$ where $\mathrm{C}$ is the number of categories

$270\left\{\left(X_{i}, Y_{i}\right) \rightarrow c_{j i}\right\}_{i}=1,0 \leq j \leq C$

\section{Output:}

272 The image representation $\mathrm{U}=\left[\mathrm{u}_{1}, \mathrm{u}_{2}, \ldots, \mathrm{u}_{\mathrm{n}}\right]$, text representation $\mathrm{V}=\left[\mathrm{v}_{1}, \mathrm{v}_{2}, \ldots ., \mathrm{v}_{\mathrm{n}}\right]$, hyper

273 parameter $\boldsymbol{\theta}_{\mathbf{x}}$ of image modality, hyper parameter $\boldsymbol{\theta}_{\mathbf{y}}$ of text modality, a common sub-space B.

\section{Initialization}

$275 \theta_{\mathrm{x}}=0.1, \theta_{y}=0.1$, batch size $=128, \eta=0.1, \gamma=0.1, \mathrm{I}_{\mathrm{x}}=\left\lceil\frac{\mathrm{n}}{\text { batch size }}\right\rceil, \mathrm{I}_{\mathrm{y}}=\left\lceil\frac{\mathrm{n}}{\text { batch size }}\right\rceil$ where $\mathrm{n}$ is

276 number of training data points.

$277 \mathrm{~S}_{\mathrm{ij}}=1$ if $\left(\mathrm{X}_{\mathrm{i}}, \mathrm{Y}_{\mathrm{i}}\right) \in\left[\mathrm{z}_{1 \mathrm{i}}, \mathrm{z}_{2 \mathrm{i}}, \ldots, \mathrm{z}_{\mathrm{Ci}}\right] 1 \leq \mathrm{j} \leq \mathrm{C}$

$278 \mathrm{~S}_{\mathrm{ij}}=0$ otherwise 
279

280 [Image Modality]

281 for iteration $=1$ to $\mathrm{I}_{\mathrm{x}}$

282

283

284

285

286

287

288

289

290

291

292

293

294

295

296

297

298

299

300

301

302

303

304

305 sub-space. (Eq. (19)) descent,

end for space.

Step-1 Select 128 data points (batch size) from Image $\mathrm{X}$ and Word Vector $\mathrm{Y}$

Step-2 Calculate learned image feature $U_{i}=f\left(X_{i}, \theta_{x}\right)$ by forward propagation

Step-3 Calculate the learned text feature $V_{i}=g\left(Y_{i}, \theta_{y}\right)$ by forward propagation

Step-4 Calculate the discrimination loss in the label space. (Eq. (18))

Step-5 Calculate the discrimination loss of both text and image representation in the common

Step-6 Calculate the modality wise invariance loss. [Eq. (23)]

Step-7 Update the linear classifier $\mathrm{C}$ parameters by minimizing the cost function

$$
\mathrm{C}=\left(\mathrm{UU}^{\mathrm{T}}\right)^{-1} \mathrm{U}^{\mathrm{T}} \mathrm{S}+\left(\mathrm{VV}^{\mathrm{T}}\right)^{-1} \mathrm{~V}^{\mathrm{T}} \mathrm{S}
$$

Step-8 Update the parameters of image network and text network using stochastic gradient

$$
\theta_{\mathrm{x}}=\theta_{\mathrm{x}}-\eta \frac{\partial \mathrm{J}}{\partial \theta_{\mathrm{x}}} \text { and } \theta_{\mathrm{y}}=\theta_{\mathrm{y}}-\eta \frac{\partial \mathrm{J}}{\partial \theta_{\mathrm{y}}}
$$

\section{[a] Calculate the discrimination loss in the label space.}

Once the features are learned from image and text modality, a linear classifier $\mathrm{C}$ is connected to image and text sub networks, which predicts the semantic labels of the projected features. This predicted label should preserve the semantic similarity with label space. The discrimination loss in the label space is calculated by $\mathrm{J}_{1}$ using the following equation:

$$
\mathrm{J}_{1}=\frac{1}{\mathrm{n}}\left\|\mathrm{C}^{\mathrm{T}} \mathrm{U}-\mathrm{S}\right\|_{\mathrm{F}}+\frac{1}{\mathrm{n}}\left\|\mathrm{C}^{\mathrm{T}} \mathrm{V}-\mathrm{S}\right\|_{\mathrm{F}}
$$

Where, $\|\cdot\|_{F}$ is Frobenius norm and $n$ is the number of instances.

\section{[b] Calculate the discrimination loss of both text and image modality in the common sub-}

The inter-modal similarity is further preserved by minimizing discrimination loss from image and text representation in the common sub-space, as denoted by Eq. (19).

$$
\mathrm{J}_{2}=\frac{1}{\mathrm{n}}\left[-\sum_{\mathrm{i}, \mathrm{j}=1}^{\mathrm{n}}\left[\mathrm{S}_{\mathrm{ij}} \theta_{\mathrm{ij}}-\log \left(1+\theta_{\mathrm{ij}}\right)\right]+\frac{1}{\mathrm{n}}\left[-\sum_{\mathrm{i}, \mathrm{j}=1}^{\mathrm{n}}\left[\mathrm{S}_{\mathrm{ij}} \varphi_{\mathrm{ij}}-\log \left(1+\varphi_{\mathrm{ij}}\right)\right]+\frac{1}{\mathrm{n}}\left[-\sum_{\mathrm{i}, \mathrm{j}=1}^{\mathrm{n}}\left[\mathrm{S}_{\mathrm{ij}} \phi_{\mathrm{ij}}-\log \left(1+\phi_{\mathrm{ij}}\right)\right]\right]\right.\right.
$$

306 The first part of Eq. (19), preserves the semantic similarity between image representation $U$ and 307

308 text representation $\mathrm{V}$ with similarity matrix $\mathrm{S}$, which is denoted as,

$$
\theta_{\mathrm{ij}}=\mathrm{U}_{* \mathrm{i}}^{\mathrm{T}} \mathrm{V}_{* \mathrm{j}}
$$

309 The above equation should maximize the likelihood

$$
\begin{aligned}
\mathrm{P}\left(\mathrm{S}_{\mathrm{ij}} \mid \mathrm{U}_{* \mathrm{i}}, \mathrm{V}_{* \mathrm{j}}\right) & =\sigma\left(\theta_{\mathrm{ij}}\right) & \text { when } \mathrm{S}_{\mathrm{ij}}=1 \\
& =1-\sigma\left(\theta_{\mathrm{ij}}\right) & \text { when } \mathrm{S}_{\mathrm{ij}}=0
\end{aligned}
$$


310 Where $\sigma\left(\theta_{\mathrm{ij}}\right)=\frac{1}{1+\mathrm{e}^{-\theta_{\mathrm{ij}}}}$ is a sigmoid function that exists between 0 to 1 , and it is preferable when

311 there is a need to predict the probability as an output. Since the probability of anything exists

312 between a range of 0 to 1 , sigmoid is the right choice.

313 It is represented as,

$$
\begin{aligned}
\mathrm{P}\left(\mathrm{S}_{\mathrm{ij}} \mid \mathrm{U}_{* \mathrm{i}}, \mathrm{V}_{* \mathrm{j}}\right) & =\pi\left(\sigma\left(\theta_{\mathrm{ij}}\right)\right)^{\mathrm{S}_{\mathrm{ij}}}\left(1-\sigma\left(\theta_{\mathrm{ij}}\right)\right)^{1-\mathrm{S}_{\mathrm{ij}}} \\
& =\sum_{\mathrm{i}, \mathrm{j}=1}^{\mathrm{n}}\left[\mathrm{S}_{\mathrm{ij}} \theta_{\mathrm{ij}}+\log \left(1-\theta_{\mathrm{ij}}\right)\right]
\end{aligned}
$$

314 Eq. (21) can be rewritten as below cost function which forces representation $\theta_{\mathrm{ij}}$ to be larger when $315 \mathrm{~S}_{\mathrm{ij}}=1$ and vice versa.

316

$$
J=-\sum_{i, j=1}^{n}\left[S_{i j} \theta_{i j}-\log \left(1+\theta_{i j}\right)\right]
$$

317 So, here cost function forces $\theta_{\mathrm{ij}}$ to be larger when $\mathrm{S}_{\mathrm{ij}}=1$ and vice versa.

318 The second part and third part of the equation measures the similarities with image 319 representation and text representations.

320

321

322

$\phi_{\mathrm{ij}}$ is text representation, for instance, $\mathrm{i}$ and $\mathrm{j}$.

$$
\varphi_{\mathrm{ij}}=\mathrm{U}_{* \mathrm{i}}^{\mathrm{T}} \mathrm{U}_{* \mathrm{j}}
$$

$\varphi_{\mathrm{ij}}$ is image representation, for instance, $\mathrm{i}$ and $\mathrm{j}$ whereas

$$
\phi_{\mathrm{ij}}=\mathrm{V}_{* \mathrm{i}}^{\mathrm{T}} \mathrm{V}_{* \mathrm{j}}
$$

\section{[c] Calculate the modality wise invariance loss}

$$
\mathrm{J}_{3}=\frac{1}{\mathrm{n}}\|\mathrm{U}-\mathrm{V}\|_{\mathrm{F}}
$$

325

The final objective function is,

$$
\mathrm{J}=\mathrm{J}_{1}+\lambda \mathrm{J}_{2}+\eta \mathrm{J}_{3}
$$

326

327

328

329

330

331

332

333

334

335

336

337

The final objective function of IDMR in Eq. (24) can be optimized during the stochastic gradient descent algorithm. The $\lambda$ and $\eta$ are hyper parameters. The $\mathrm{J}_{1}, \mathrm{~J}_{2}$, and $\mathrm{J}_{3}$ are the loss functions, used to preserve inter-modal similarity between image and text modality. The proposed framework has used the sigmoid activation function, which is a nonlinear function used to learn complex structures in the data. However, sometimes it suffers from vanishing gradient descent, which prevents deep networks to learn from learning effectively. The problem of vanishing gradient can be solved by using another activation function, like rectified linear activation unit(ReLU).

\section{Results}

To evaluate the effectiveness of the proposed framework, we have performed experiments on well-known datasets MSCOCO (Lin et al., 2015b), Flickr8k ("Flickr8K"), and XMedia("PKU XMediaNet Dataset"; Zhai, Peng \& Xiao, 2014; Peng et al., 2016), which are widely used in the 
338

339

340

341

342

343

344

345

346

347

348

349

350

351

352

353

354

355

356

357

358

359

360

361

362

363

364

365

366

367

368

369

370

371

372

\section{3}

374

375

376

377

studies. The MSCOCO dataset has total of 3,28,000 images, which is divided into 91 categories and each image is associated with at least 5 captions. The MSCOCO dataset consists of daily scene images and their descriptions. The training set consists of 15,000 images and the query set consists of 4000 images. The Flickr8k dataset contains 8000 images and each paired with 5 different captions. The training set consists of 6000 images and the testing set consists of 1000 images. The XMedia dataset has text, image, video, and audio modality, which has a total of 20 different categories and each category has 600 media instances. The training set consists of 8000 images and the testing set consists of 1500 images. We perform experiments on GPU architecture, NVIDIA cuda cores-3840, the memory size of 12 GB GDDR5X, 32 GB RAM, 2TB hard disk, and Intel Core $178^{\text {th }}$ generation. We have considered mean Average Precision (mAP) as a statistical measure, which is used to measure the performance of the CMR system (Peng et al., 2017; Yanagi et al., 2020).

Following is the analysis after performing experiments.

1. The biggest challenge in text modality is to preserve semantic similarity between different words. Here experiments are carried out between different vectorization methods like BoW, CBOW, SG, and Glove. The syntactic and semantic pairs are selected from each Multi-Modal dataset like MSCOCO, Flickr8k, and XMedia. The cosine similarity is used to calculate the similarity between different pairs of words. Figure 4 shows the performance of various vectorization methods on Multi-Modal datasets. The experiment shows that Glove outperforms other vectorization methods. The Glove achieves better performance as it can preserve the similarity of words by considering the entire corpus. Due to better performance, Glove is selected as a vectorization method in the proposed framework.

2. The objective function of IDMR generates real-valued representation of image and text modality in the common sub-space, which preserves inter-modal and intra-modal similarity. The performance of IDCMR is compared with state-of-the-art CMR methods. Source codes of DCMH, DSCMR, SePH, SCM, DBM, and DAE are provided by the corresponding authors. Figure 5, Figure 6, Figure 7, and Figure 8 show the performance of IDCMR on MSCOCO and XMedia dataset for image $\rightarrow$ text and text $\rightarrow$ image retrieval, respectively. The experiment shows that IDCMR outperforms over state-of-the-art methods in both image $\rightarrow$ text and text $\rightarrow$ image retrieval. The advantage of IDCMR over other state-of-the-art methods is that the objective function of IDCMR preserves both inter-modal similarity and intra-modal similarity.

\section{Conclusions}

The work presented in the paper has proposed a framework called "Improvement of Deep CrossModal Retrieval (IDCMR)", which is restricted to image and text modality. The generated heterogeneity gap is bridged by generating a common sub-space. The nature of the common subspace is real-valued, which preserves similarities between different modalities. The uniqueness

PeerJ Comput. Sci. reviewing PDF | (CS-2020:10:54155:1:1:NEW 24 Feb 2021) 
378 of our proposed framework is that we consider both the inter-modal and intra-modal similarities 379 between various modalities. The proposed framework outperforms state-of-the-art methods in 380 text $\rightarrow$ image and image $\rightarrow$ text retrieval tasks on Multi-Modal datasets. However, there exist many 381 types of noise and redundancies in Multi-Modal data, which need to be resolved to improve the 382 performance of the CMR system. Here the proposed framework is restricted to image and text 383 modality, which can be extended to other modalities.

384

385

386

387

388

389

390

391

392

393

394

395

396

397

398

399

400

401

402

403

404

405

406

407

408

409

410

411

412

413

\section{References}

Ding G, Guo Y, Zhou J. 2014. Collective Matrix Factorization Hashing for Multimodal Data. In: 2014 IEEE Conference on Computer Vision and Pattern Recognition. Columbus, OH, USA: IEEE, 2083-2090. DOI: 10.1109/CVPR.2014.267.

Flickr8K. Available at https://kaggle.com/shadabhussain/flickr8k (accessed October 30, 2020).

Hardoon DR, Szedmak S, Shawe-Taylor J. 2004. Canonical Correlation Analysis: An Overview with Application to Learning Methods. Neural Computation 16:2639-2664. DOI: 10.1162/0899766042321814.

Hongtao L, Qinchuan Z. 2016. Applications of Deep Convolutional Neural Network in Computer Vision. Journal of Data Acquisition and Processing.

Jiang Q-Y, Li W-J. 2016. Deep Cross-Modal Hashing. arXiv:1602.02255 [cs].

Kumar S, Udupa R. 2011. Learning hash functions for cross-view similarity search. In: Proceedings of the Twenty-Second international joint conference on Artificial Intelligence Volume Volume Two. IJCAI'11. Barcelona, Catalonia, Spain: AAAI Press, 1360-1365.

Lin Z, Ding G, Mingqing Hu, Wang J. 2015a. Semantics-preserving hashing for cross-view retrieval. In: 2015 IEEE Conference on Computer Vision and Pattern Recognition (CVPR). Boston, MA, USA: IEEE, 3864-3872. DOI: 10.1109/CVPR.2015.7299011.

Lin T-Y, Maire M, Belongie S, Bourdev L, Girshick R, Hays J, Perona P, Ramanan D, Zitnick CL, Dollár P. 2015b. Microsoft COCO: Common Objects in Context. arXiv:1405.0312 [cs].

Lowe DG. 2004. Distinctive Image Features from Scale-Invariant Keypoints. International Journal of Computer Vision 60:91-110. DOI: 10.1023/B:VISI.0000029664.99615.94.

Mikolov T, Chen K, Corrado G, Dean J. 2013a. Efficient Estimation of Word Representations in Vector Space. arXiv:1301.3781 [cs].

Mikolov T, Sutskever I, Chen K, Corrado G, Dean J. 2013b. Distributed Representations of Words and Phrases and their Compositionality. arXiv:1310.4546 [cs, stat].

Ngiam J, Khosla A, Kim M, Nam J, Lee H, Ng AY. 2011. Multimodal Deep Learning. :8.

Peng Y, Zhai X, Zhao Y, Huang X. 2016. Semi-Supervised Cross-Media Feature Learning With Unified Patch Graph Regularization. IEEE Transactions on Circuits and Systems for Video Technology 26:583-596. DOI: 10.1109/TCSVT.2015.2400779. 
414 Peng Y, Zhu W, Zhao Y, Xu C, Huang Q, Lu H, Zheng Q, Huang T, Gao W. 2017. Cross-media 415 analysis and reasoning: advances and directions. Frontiers of Information Technology \& 416 Electronic Engineering 18:44-57. DOI: 10.1631/FITEE.1601787.

417 Pennington J, Socher R, Manning C. 2014. Glove: Global Vectors for Word Representation. In: 418 Proceedings of the 2014 Conference on Empirical Methods in Natural Language Processing 419 (EMNLP). Doha, Qatar: Association for Computational Linguistics, 1532-1543. DOI: 420 10.3115/v1/D14-1162.

421 PKU XMediaNet Dataset. Available at http://59.108.48.34/tiki/XMediaNet/ (accessed October $42230,2020)$.

423 Song J, Yang Y, Yang Y, Huang Z, Shen HT. 2013. Inter-media hashing for large-scale retrieval 424 from heterogeneous data sources. In: Proceedings of the 2013 ACM SIGMOD International 425 Conference on Management of Data. SIGMOD '13. New York, NY, USA: Association for 426 Computing Machinery, 785-796. DOI: 10.1145/2463676.2465274.

427 Srivastava N, Salakhutdinov R. 2014. Multimodal learning with deep Boltzmann machines. The 428 Journal of Machine Learning Research 15:2949-2980.

429 Van Loan CF. 1976. Generalizing the Singular Value Decomposition. SIAM Journal on 430 Numerical Analysis 13:76-83. DOI: 10.1137/0713009.

431 Wang W, Yang X, Ooi BC, Zhang D, Zhuang Y. 2016a. Effective deep learning-based multi432 modal retrieval. The VLDB Journal 25:79-101. DOI: 10.1007/s00778-015-0391-4.

433 Wang K, Yin Q, Wang W, Wu S, Wang L. 2016b. A Comprehensive Survey on Cross-modal 434 Retrieval. arXiv:1607.06215 [cs].

435 Weiss Y, Torralba A, Fergus R. 2009. Spectral Hashing. In: Koller D, Schuurmans D, Bengio Y, 436 Bottou L eds. Advances in Neural Information Processing Systems 21. Curran Associates, Inc., $437 \quad 1753-1760$.

438 Xu G, Li X, Zhang Z. 2020. Semantic Consistency Cross-Modal Retrieval With Semi439 Supervised Graph Regularization. IEEE Access. DOI: 10.1109/ACCESS.2020.2966220.

440 Yanagi R, Togo R, Ogawa T, Haseyama M. 2020. Enhancing Cross-Modal Retrieval Based on 441 Modality-Specific and Embedding Spaces. IEEE Access 8:96777-96786. DOI:

442 10.1109/ACCESS.2020.2995815.

443 Zhai X, Peng Y, Xiao J. 2014. Learning Cross-Media Joint Representation With Sparse and 444 Semisupervised Regularization. IEEE Transactions on Circuits and Systems for Video 445 Technology 24:965-978. DOI: 10.1109/TCSVT.2013.2276704.

446 Zhang D, Li W-J. 2014. Large-scale supervised multimodal hashing with semantic correlation 447 maximization. In: Proceedings of the Twenty-Eighth AAAI Conference on Artificial 448 Intelligence. AAAI'14. Québec City, Québec, Canada: AAAI Press, 2177-2183. 
449 Zhen L, Hu P, Wang X, Peng D. 2019. Deep Supervised Cross-Modal Retrieval. 2019

450 IEEE/CVF Conference on Computer Vision and Pattern Recognition (CVPR). DOI:

451 10.1109/CVPR.2019.01064.

452 Zhou J, Ding G, Guo Y. 2014. Latent semantic sparse hashing for cross-modal similarity search. 453 DOI: 10.1145/2600428.2609610. 
Figure 1

Image-Text pairs from MSCOCO and Xmedia dataset 


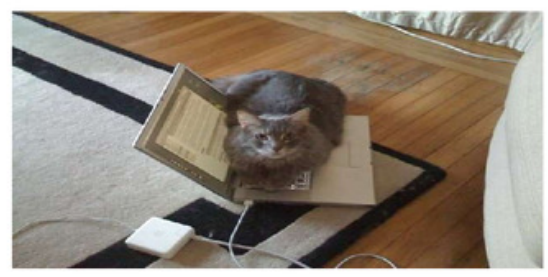

(A)

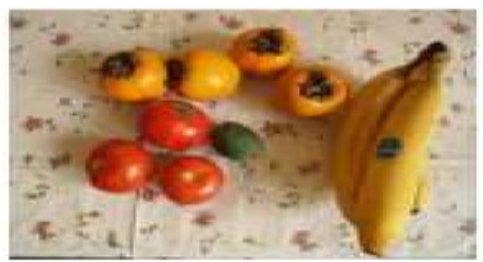

(B)

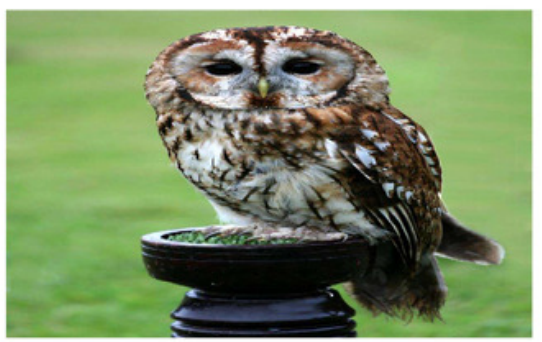

(C)

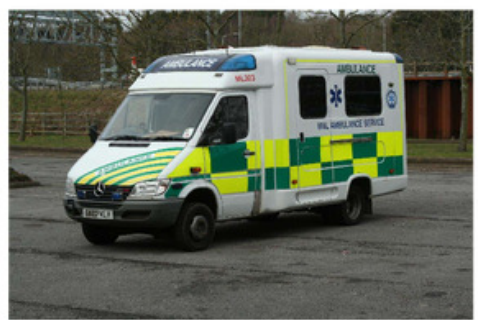

(D) 
Figure 2

Description of CBOW and SG

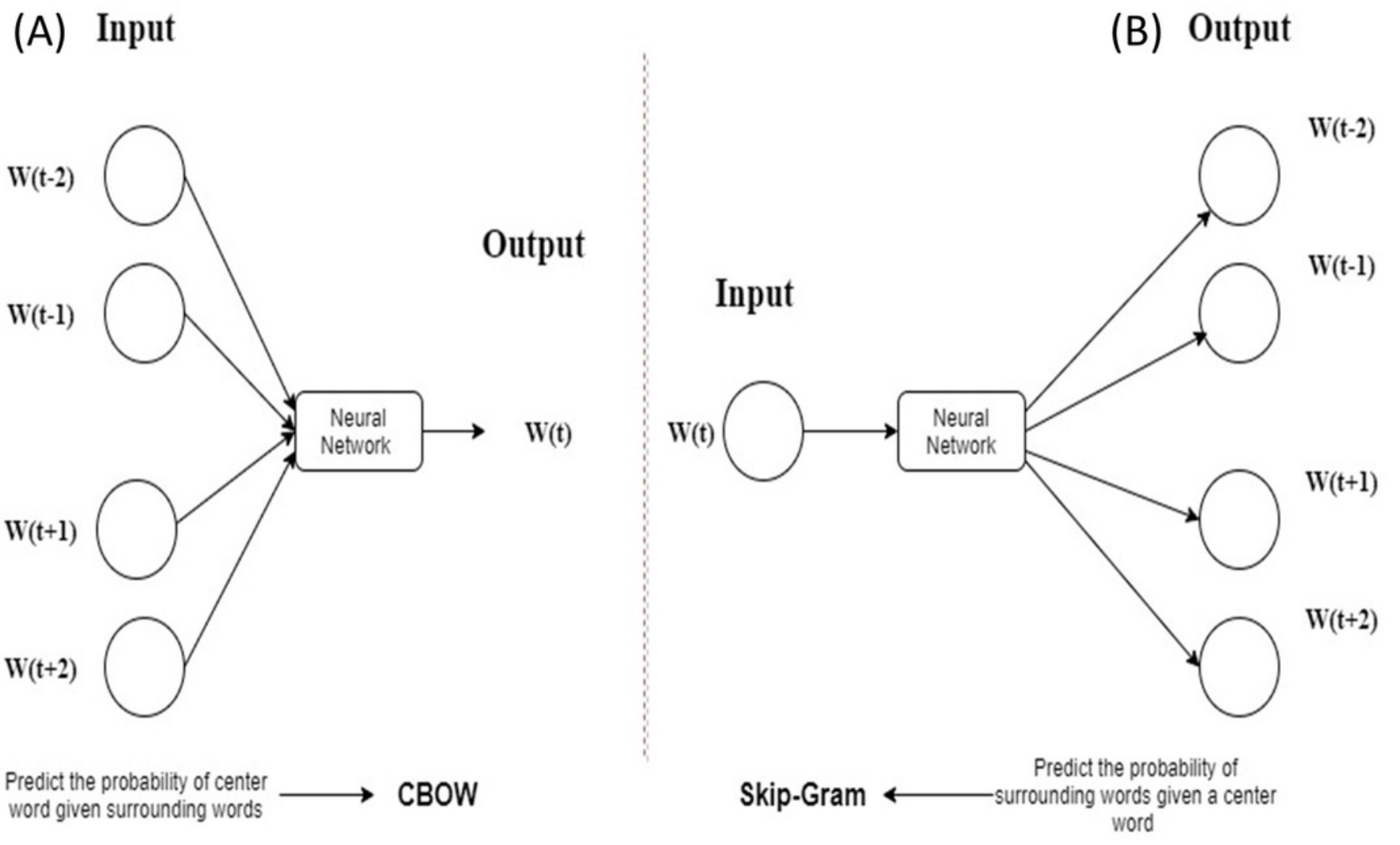


Figure 3

Proposed Framework (IDCMR)

\begin{tabular}{|l|l|l|l|l|l|l|l|}
\hline Conv1 & Conv2 & Conv3 & Conv4 & Conv5 & fc6 & fc7 & fc8 \\
\hline f:64 $611 \times 11$ & $\mathrm{f}: 265 \times 5 \times 5$ & $\mathrm{f}: 265 \times 3 \times 3$ & $\mathrm{f}: 265 \times 3 \times 3$ & $\mathrm{f}: 265 \times 3 \times 3$ & 8192 & 4096 & 4096 \\
$\mathrm{~s}: 4$ & $\mathrm{~s}: 1$ & $\mathrm{~s}: 1$ & $\mathrm{~s}: 1$ & $\mathrm{~s}: 1$ & & & \\
$\mathrm{p}: 0$ & $\mathrm{p}: 2$ & $\mathrm{p}: 1$ & $\mathrm{p}: 1$ & $\mathrm{p}: 1$ & & & \\
$2 \times 2$ Max Pool & $2 \times 2$ Max Pool & & & $2 \times 2$ Max Pool & & & \\
\hline
\end{tabular}

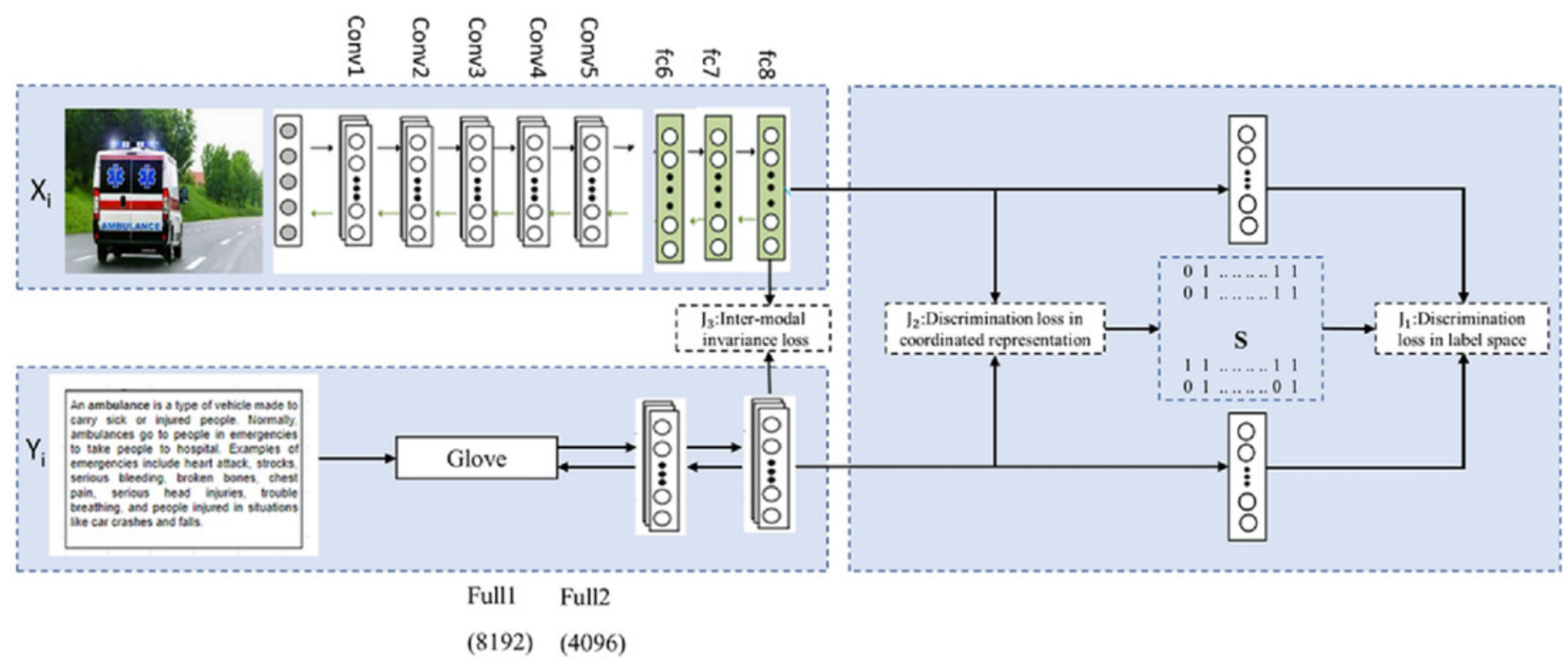


Figure 4

Performance of Vectorization Methods on Multi-Modal Datasets 


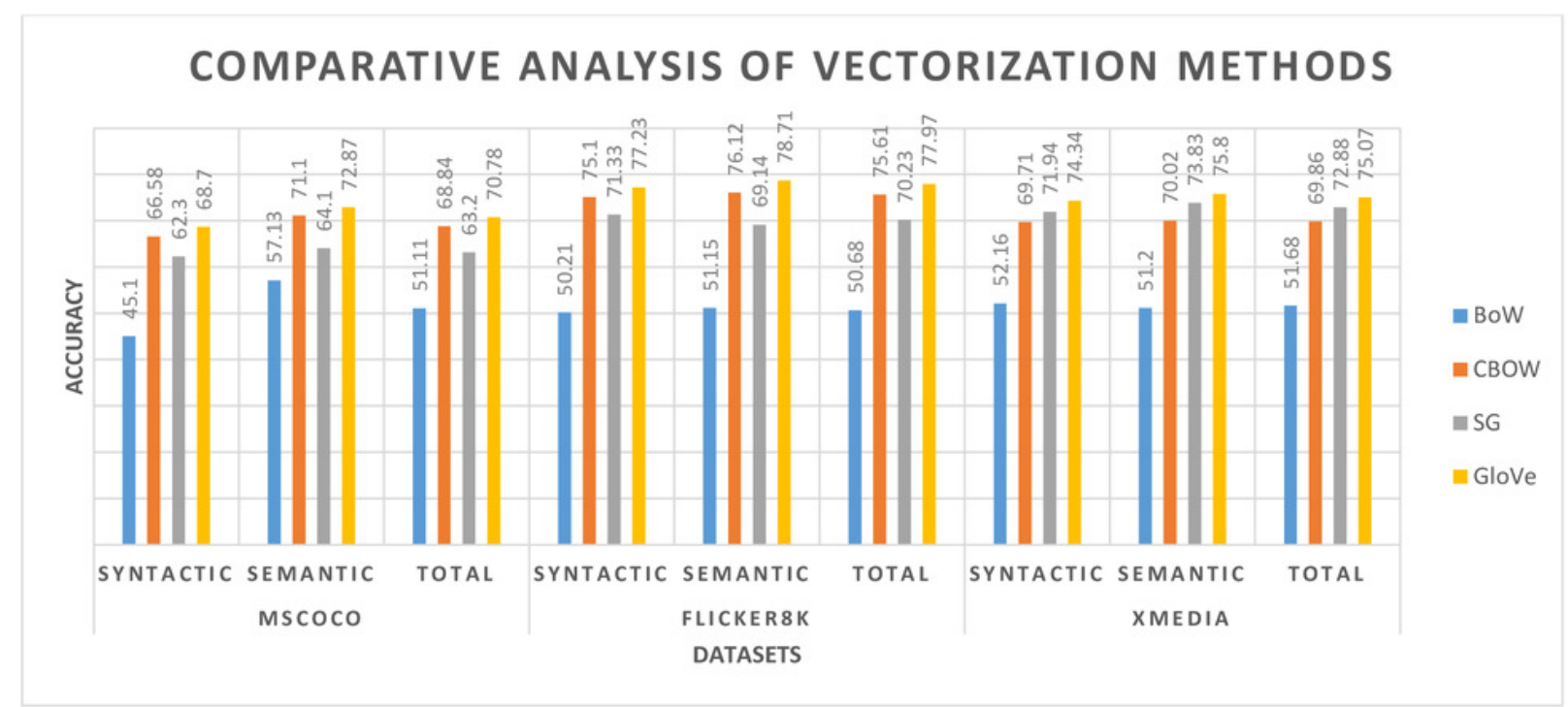


Figure 5

Performance of CMR methods on MSCOCO Dataset for Image $\rightarrow$ Text Retrieval 


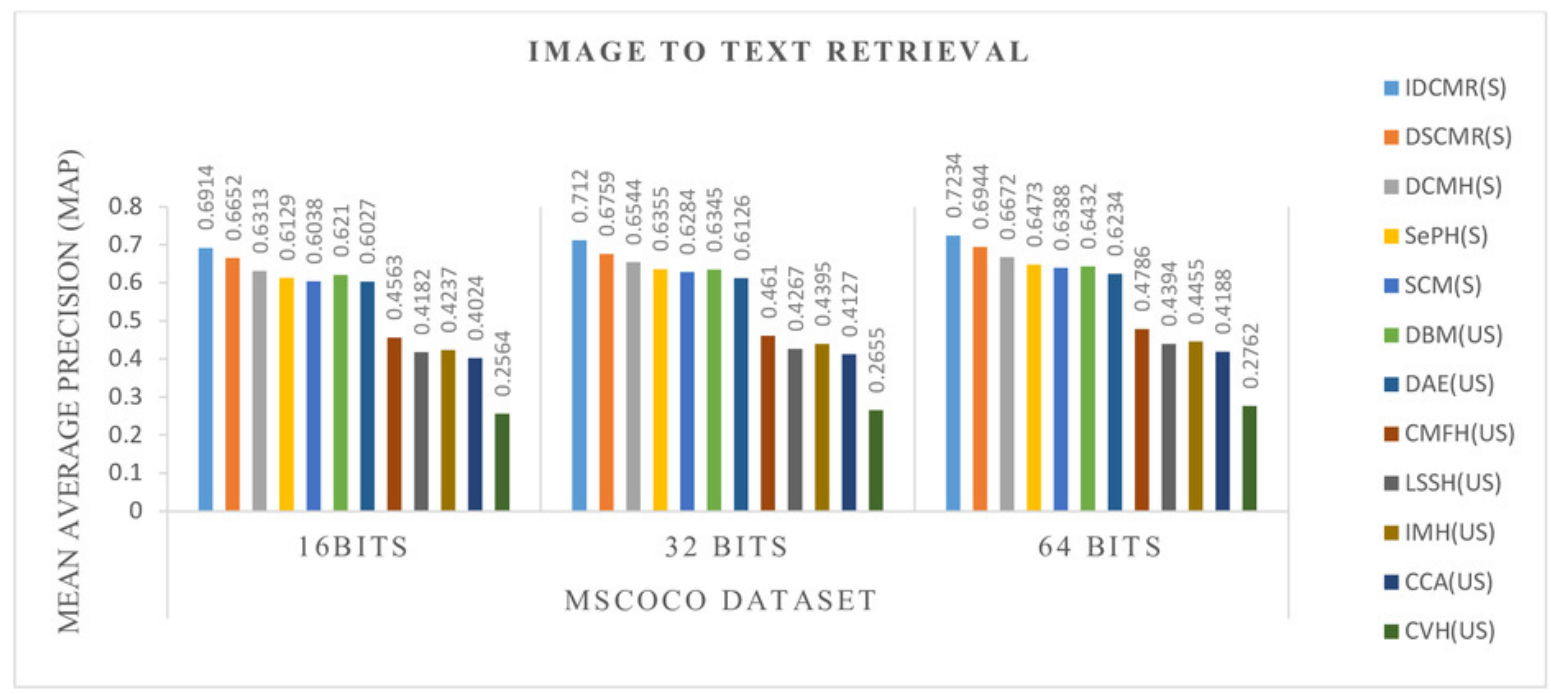


Figure 6

Performance of CMR methods on Xmedia Dataset for Image $\rightarrow$ Text Retrieval 


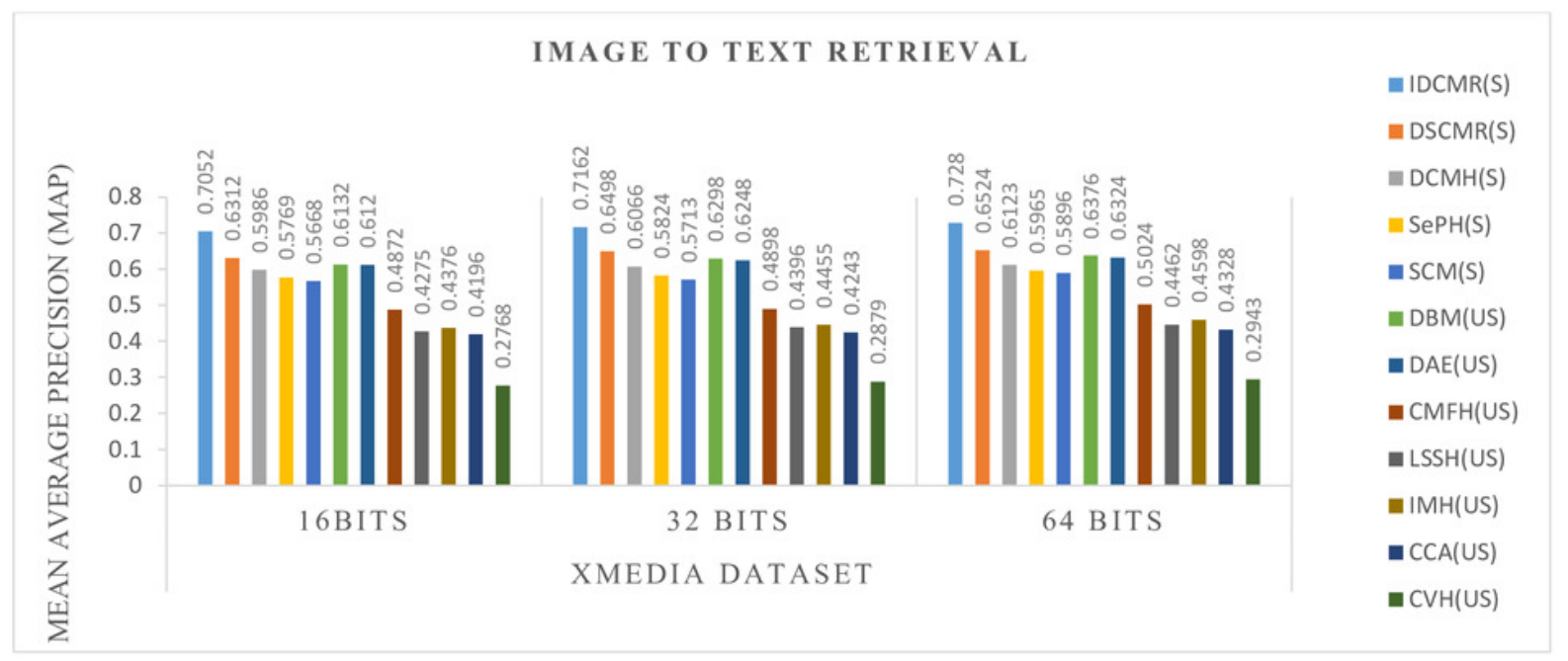


Figure 7

Performance of CMR methods on MSCOCO Dataset for Text $\rightarrow$ Image Retrieval 


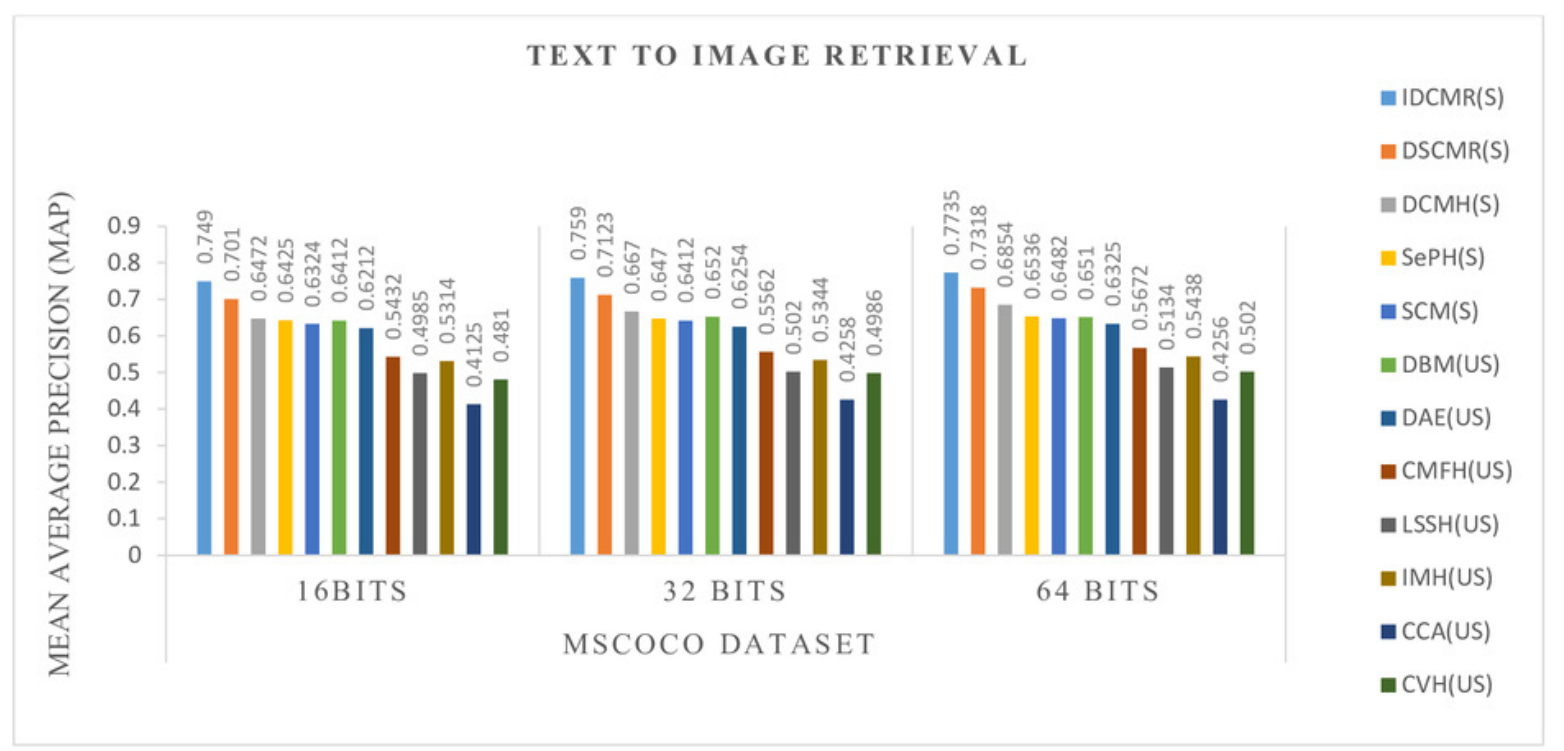


Figure 8

Performance of CMR methods on Xmedia Dataset for Text $\rightarrow$ Image Retrieval 


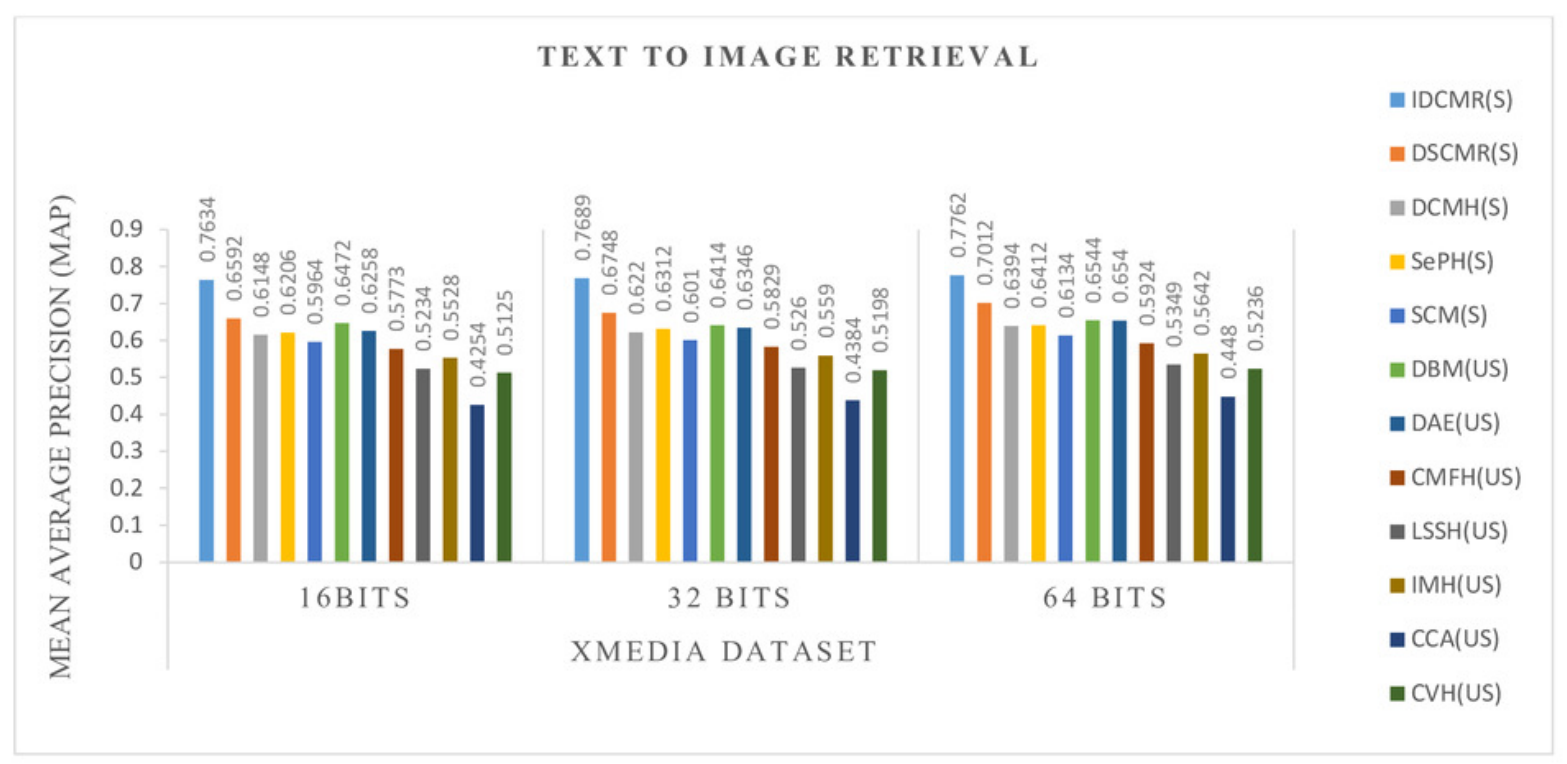

